\title{
Estudio morfológico de Naiadinium polonicum (Dinophyceae) reportado por primera vez en aguas continentales de Chile
}

\section{Morphological study of Naiadinium polonicum (Dinophyceae) reported for the first time in Chilean inland waters}

\author{
Enrique Ascencio ${ }^{1 *}$, Patricio Rivera ${ }^{1},{\text { Fabiola } \text { Cruces }^{1} \& \text { Irma Vila }}^{2}$ \\ ${ }^{1}$ Departamento de Botánica, Facultad de Ciencias Naturales y Oceanográficas, Universidad de Concepción, Casilla 160-C, \\ Concepción, Chile. \\ 2Departamento de Ciencias Ecológicas, Facultad de Ciencias, Universidad de Chile, Casilla 653, Santiago, Chile. \\ *eaascenc@gmail.com; eascencio@udec.cl
}

\begin{abstract}
RESUMEN
Naiadinium polonicum es un dinoflagelado ampliamente distribuido en lagos y embalses del Hemisferio Norte y en ocasiones asociado a la mortandad de peces por la producción de toxinas. Durante mayo de 2013 se detectó una proliferación

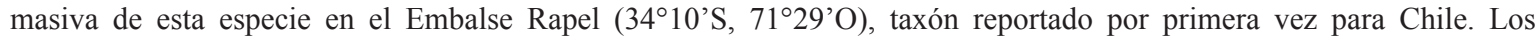
caracteres morfológicos de los ejemplares analizados mediante técnicas de microscopia óptica y electrónica de barrido son concordantes con las descripciones e ilustraciones conocidas en la literatura para la especie. Interpretamos a Naiadinium polonicum como un taxón conespecífico con Naiadinium biscutelliforme y consideramos a la especie variable con respecto al número y tamaño relativo de las placas intercalares anteriores (1-2a). Naiadinium polonicum alcanzó una concentración máxima de 2572 células $/ \mathrm{mL}$, y dado que este fenómeno sólo fue observado en una ocasión, atribuimos preliminarmente a las elevadas cantidades de nutrientes $\left(66 \mu \mathrm{gL}^{-1} \mathrm{PT}\right.$ y $\left.645.67 \mu \mathrm{gL}^{-1} \mathrm{NT}\right)$ disponibles en la columna de agua como el factor que probablemente originó la proliferación.
\end{abstract}

Palabras clave: Dinoflagelados dulceacuícolas, Peridinium polonicum, proliferación algal.

\begin{abstract}
Naiadinium polonicum is a dinoflagellate widely distributed in lakes and reservoirs of the Northern Hemisphere, sometimes associated with fish mortality by production of toxins. During May 2013 a massive proliferation of this species was detected in Rapel Reservoir (3410'S, $\left.71^{\circ} 29^{\prime} \mathrm{W}\right)$. In the present paper a morphological study of the species was performed using light and electron microscopical techniques. This is the first record of $N$. polonicum for Chilean continental waters. The morphological characters of the specimens analyzed are consistent with the descriptions and illustrations found in the literature. In this study, Naiadinium polonicum was considered as a conspecific taxon to Naiadinium biscutelliforme, and we considered the species to be variable with relation to the number and relative size of the intercalary plates (1-2a). Naiadinium polonicum reached a maximum concentration of $2572 \mathrm{cells} / \mathrm{mL}$, and since this phenomenon was observed only once we preliminary attributed the high content of nutrients $\left(66 \mu \mathrm{gL}^{-1} \mathrm{PT}, 645.67 \mu \mathrm{gL}^{-1} \mathrm{NT}\right)$ available in the water column as the preponderant factor that probably caused the proliferation.
\end{abstract}

KeYwords: Freshwater dinoflagellates, Peridinium polonicum, bloom.

\section{INTRODUCCIÓN}

Los dinoflagelados son generalmente un componente minoritario de las comunidades fitoplanctónicas de los ecosistemas acuáticos continentales. Sin embargo, bajo el efecto combinado de diversas condiciones ambientales (e.g. altas disponibilidades de nitrógeno y fósforo, aumento de la temperatura, entre otras), algunas especies pueden originar proliferaciones masivas (Reynolds 2006, Almanza et al. 2016a). Entre ellas destacan especies de los géneros Ceratium, Peridiniopsis y Peridinium, reportándose asociado a dichos eventos numerosos impactos ecosistémicos, económicos y sanitarios (Roset et al. 2002, Carty 2014, Almanza et al. 2016a).

Durante mayo de 2013 se detectó en el Embalse Rapel un desarrollo masivo de Naiadinium polonicum (Wołoszyńska) Carty. Esta especie ha producido proliferaciones en distintos países del Hemisferio Norte (Adachi 1965, Nolen et al. 
1989, Roset et al. 2002), y en algunos casos fue relacionada a la producción de toxinas de distinta naturaleza, las cuales originaron mortandad de peces en Japón (Hashimoto et al. 1968, Oshima et al. 1989) y España (Roset et al. 2002). Hasta el presente, Naiadinium polonicum es la única especie de dinoflagelado de agua dulce relacionada a la producción de toxinas con efectos nocivos en vertebrados (WHO 2003, Zaccaroni \& Scaravelli 2008, Carty 2014). En otras especies se han descrito sustancias tóxicas, cuyos efectos son alelopáticos, defensa o de inhibición de competidores sobres otros componentes del fitoplancton (Wu et al. 1998, Rengefors \& Legrand 2001).

El género Naidinium Carty fue propuesto para albergar dos especies interpretadas anteriormente como Peridinium o Peridiniopsis, pero cuya fórmula tabular no se ajusta con ninguno de ellos (Carty 2014). La especie tipo, N. polonicum, descrita originalmente como Peridinium polonicum fue ilustrada con sólo una placa intercalar anterior (Wołoszyńska 1916: Pl. 12, figs. 6, 2) y posteriormente transferida al género Peridiniopsis (Bourrelly 1968). Glenodinium gymnodinium Penard, descrito a partir de material proveniente del lago Geneva (Europa central) y a pesar que sus ilustraciones no exhiben tabulación (Penard 1891: Pl. 4, figs. 8-10), es reconocida como sinónimo de $P$. polonicum por distintos autores (Lindemann 1925, Lefèvre 1932, Schiller 1937, Kiselev 1954, Matvienko \& Litvinenko 1977, Popovský \& Pfiester 1990, Carty 2014, Craveiro et al. 2015), en tanto otros la consideran como una especie independiente (Huber-Pestalozzi 1950, Bourrelly 1970, Starmach 1974). Naiadinium biscutelliforme (Thompson) Carty, la segunda especie del género según Carty (2014) fue descrita como Glenodinium gymnodinium Penard var. biscutelliforme Thompson e ilustrada con dos placas intercalares anteriores de similar tamaño (Thompson 1951: p. 281, figs. 35, 37).

En el presente trabajo se reporta por primera vez la presencia de Naiadinium polonicum en Chile. Los ejemplares encontrados en una proliferación masiva en el Embalse Rapel fueron analizados mediante técnicas de microscopia óptica y electrónica de barrido y se estudió la variación del número de placas intercalares anteriores, proponiéndose la sinonimia de $N$. biscutelliforme como un taxón conespecífico de $N$. polonicum.

\section{MATERIALES Y MÉTODOS}

ÁreA DE ESTUdio

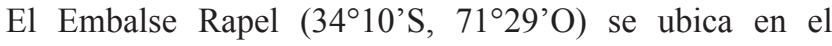
límite de las provincias de Cardenal Caro y Cachapoal, en Chile central (Región del Libertador General Bernardo O'Higgins), a $70 \mathrm{~m}$ sobre el nivel del mar (Vila et al. 2000). El embalse fue construido en 1968 para la generación de energía eléctrica y desde su creación la calidad del agua ha experimentado un rápido deterioro. Alrededor de su cuenca se desarrollan múltiples actividades agropecuarias, mineras y turísticas; consecuencia de ellas, se han incorporado altas descargas de nutrientes en la columna de agua y han aumentado las concentraciones de metanos y sulfatos en sus sedimentos (Vila et al. 2000, Martínez et al. 2003), generándose un sistema con tendencia a la hipertrofia (Vila \& Pardo 2003, Dorador et al. 2007). El Embalse Rapel posee un volumen aproximado de $0,834 \mathrm{~km}^{3}$ y una profundidad máxima de $90 \mathrm{~m}$. Es un sistema monomíctico temperado y en función de sus características batimétricas e hidrológicas se han definido tres sectores (Fig. 1): las subcubetas Las Balsas y Alhué, con influencias fluviales de los ríos Cachapoal y Alhué respectivamente, y la zona de La Represa, con características lacustres. El sector Las Balsas, lugar donde fue detectada la proliferación, presenta un área de $37,4 \mathrm{~km}^{2}$, un volumen de $0,2010 \mathrm{~km}^{3}$, una profundidad máxima de $24 \mathrm{~m}$ y un tiempo de residencia de 12,4 días (Martínez et al. 2003).

\section{OBTENCIÓN DE PARÁMETROS FÍSICO-QUíMICOS}

La temperatura, el $\mathrm{pH}$, la conductividad, el oxígeno disuelto y la concentración de clorofila $a$ se midieron en superficie con una sonda multiparámetro YSY. La transparencia del agua se midió utilizando la profundidad de visibilidad

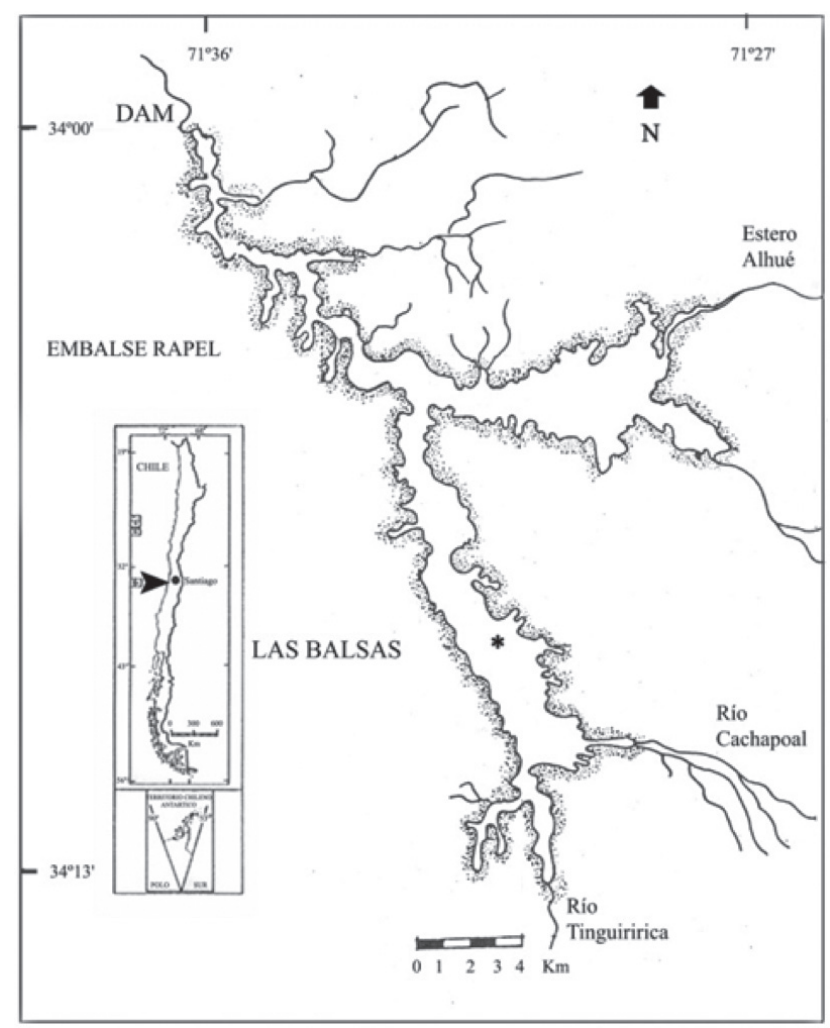

FIgURA 1: Ubicación geográfica del Embalse Rapel (Chile central, $34^{\circ} 10^{\prime} \mathrm{S}, 71^{\circ} 29^{\prime} \mathrm{O}$ ), estación de muestreo (*). / Geographic

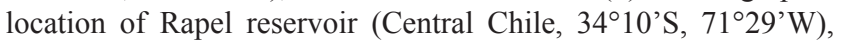
sampling station $(*)$. 
de un disco de Secchi, el fósforo total (P Total) y el nitrógeno Kjeldahl (N Total) se midieron de acuerdo con la metodología de Mühlhauser et al. (1987).

\section{RECOLECCIÓN Y ANÁLISIS DE LABORATORIO}

Para el presente estudio el material biológico se recolectó el 9 de mayo de 2013 mediante una botella plástica de 1L y los recuentos se realizaron con una cámara de precisión Sedgwick-Rafter en un microscopio invertido Olympus CK2, según la metodología de Villafañe \& Reid (1995). Para la identificación detallada del dinoflagelado se siguió la metodología descrita por Boltovskoy (1995) mediante un microscopio óptico Olympus CT-31 y un microscopio electrónico de barrido JEOL JSM-6380 LVY perteneciente al Centro de Espectroscopía y Microscopía Electrónica de la Universidad de Concepción.

\section{RESULTADOS}

Morfología de NAIAdiNiUM POLONICUM (WoŁoszyńSKA) CARTY

Naiadinium polonicum (Wołoszyńska) Carty. Figs. 2, 3A$\mathrm{K}, 4$.

Basiónimo: Glenodinium gymnodinium Penard, Bull. Trav. Soc. Bot. Genève 6: 54, P1. 4, figs. 8-10. 1891.

Sinónimos: Peridinium polonicum Wołoszyńska, Bull. Int. Acad. Sci. Cracovie, Cl. Sci. Math. Sér. B, Sci. Nat: 271, Taf. 12, figs. 1-10. 1916 [1915]. Peridiniopsis polonicum (Wołoszyńska) Bourrelly, Protistologica 4(1): 9. 1968. Peridiniopsis gymnodinium (Penard) Bourrelly, Protistologica 4(1): 9. 1968. Glenodinium gymnodinium Penard var. biscutelliforme Thompson, Lloydia 13(4): 293, figs. 35-40. 1951 [1950]. Naiadinium biscutelliforme (Thompson) Carty, Freshwater dinoflagellates of North America: 120, figs. 85-87. 2014. Naiadinium polonicum (Wołoszyńska) Carty, Freshwater dinoflagellates of North America: 123, Pl. 18, figs. 2 a-f. 2014.

Nota nomenclatural: De acuerdo al principio de prioridad (Art. 11) establecido en el Código Internacional de Nomenclatura para algas, hongos y plantas (McNeill et al. 2012), el nombre legítimo de la especie debería tener el epíteto "gymnodinium". Sin embargo, recientemente una propuesta de conservar el epíteto "polonicum" se encuentra sometido a la revista Taxon. Dicha proposición se sustenta en la presencia de iconografía tipo con ilustraciones que exhiben tabulación y en el amplio uso del epíteto por la comunidad científica debido a las proliferaciones masivas generadas por la especie, asociadas con generación de ictiotoxinas (S.C. Craveiro \& A.J. Calado, Universidad de Aveiro, comunicación personal).

Las células observadas en vista ventral poseen forma ovoide (Figs. 2, 3C-E), presentan una fuerte compresión dorso-ventral, con el lado ventral cóncavo y el lado dorsal es más o menos convexo (Fig. 3B). La longitud de las tecas osciló entre 23,2 y 43,5 $\mu \mathrm{m}(\mathrm{n}=40)$, el transdiámetro 23,2$34,8 \mu \mathrm{m}(\mathrm{n}=40)$ y el espesor entre $17,0-26,4 \mu \mathrm{m}(\mathrm{n}=40)$. La superficie de las placas está ornamentada por un reticulado irregular, el cual tiene diverso grado de desarrollo, con uno o dos poros en cada una de las areolas (Figs. 3A-K). Las bandas intercalares carecen de ornamentación superficial y pueden estar bien desarrolladas (Figs. 3I-J). La epiteca tiene forma cónica y es equivalente o levemente más alta que la hipoteca (Figs. 2, 3C-E). La fórmula tabular determinada fue: Po, X, 4', 2a, 7", 5", 2"', 6C (= 5C+t), 4S (?). El complejo de poro apical (CPA) está constituido por la placa de poro apical (Po), la que tiene contacto directo con la 3 ', $\mathrm{y}$ la placa de la canaleta $(\mathrm{X})$ que tiene forma rectangular $\mathrm{y}$ es de posición ventral (Figs. 3A-B, 3E, 3G-H). El CPA se encuentra rodeado por aletas poco desarrolladas que nacen de las placas apicales laterales (2' y 4'). El poro apical es elongado y está obturado por un "tapón" (Figs. $3 \mathrm{G}-\mathrm{H})$. En vista dorsal se observó en la epiteca dos placas intercalares anteriores de forma rectangular o cuadrada, ambas ubicadas sobre la cuarta precingular (Figs. 3A, 3E, 3I-K). En todos los casos observados la primera placa intercalar (1a) siempre fue aproximadamente 3 veces más pequeña que la segunda intercalar (Figs. 3I-K). La hipoteca tiene forma redondeada y está conformada por 7 placas (5 postcingulares y 2 antapicales) (Figs. 3C-F). En la mayoría de las tecas analizadas se observó en el antápice una espina entre la sulcal posterior (Sp), 1" y 2"'. Esta espina es una expansión de la cresta marginal de la placa 1"' (Figs. 3C-F). El cingulum está constituido por 6 placas con escasa reticulación, es ecuatorial, excavado y con un

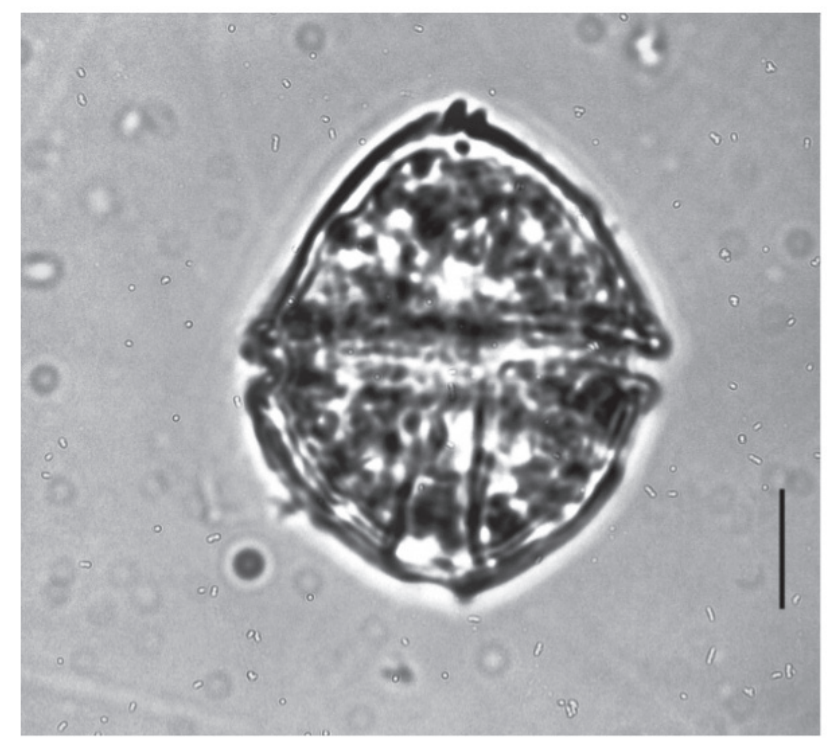

Figura 2: Naiadinium polonicum (LM). Una célula ovalada en vista ventral. Escala $=10 \mu \mathrm{m}$. / Naiadinium polonicum (LM). Ventral view of an oval cell. Scale bar $=10 \mu \mathrm{m}$. 

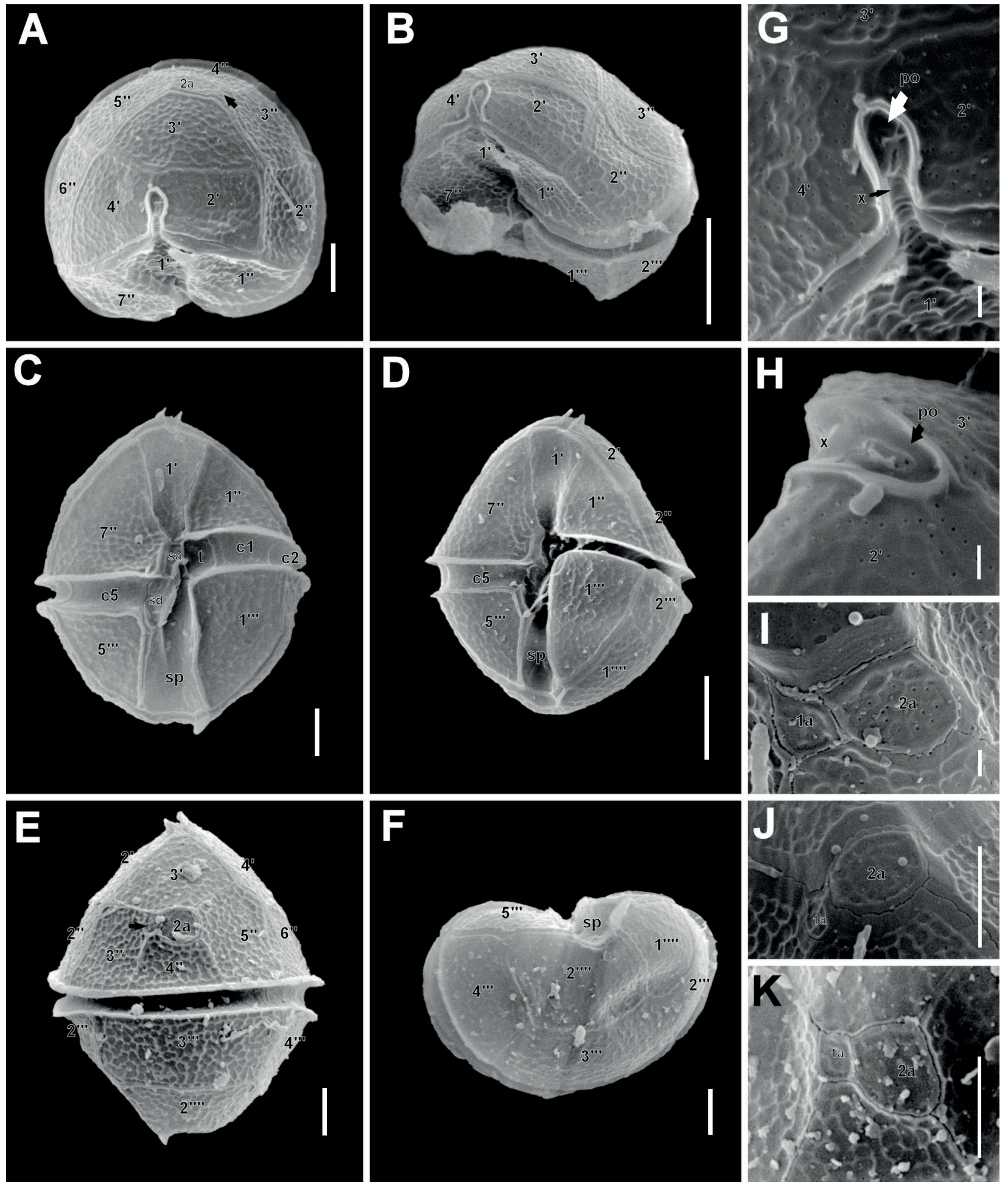

Figura 3: Naiadinium polonicum del Embalse Rapel, Chile (MEB). A) Vista apical, 1a de pequeño tamaño (flecha). B) Célula comprimida dorsoventral. C-D) Vista ventral, cingulum descendente y desplazado una altura cingular sobre el lado izquierdo. E) Vista dorsal, 1a de pequeño tamaño (flecha) y 2a de forma rectangular. F) Vista Antapical. G-H) Complejo del poro apical (Po y X). I-K) Detalles de las placas intercalares anteriores en diferentes epitecas. Escalas: B, D $=10 \mu \mathrm{m}$. A, C, E-F, J-K $=5 \mu \mathrm{m}$. G-I $=1 \mu \mathrm{m}$. / Naiadinium polonicum from Rapel reservoir, Chile (SEM). A) Apical view, 1a of small size (arrow). B) cell with compressed dorsoventrally. C-D) Ventral view, cingulum descendent and one width displacement on its right side. E) Dorsal view, 1a of small size (arrow) and 2a rectangular shape. F) Antapical view. G-H) Apical pore complex (Po and X). I-K) Detail of the anterior intercalary plates in different epithecas. Scale bars: B, D $=10 \mu \mathrm{m}, \mathrm{A}, \mathrm{C}, \mathrm{E}-\mathrm{F}, \mathrm{J}-\mathrm{K}=5 \mu \mathrm{m}, \mathrm{G}-\mathrm{I}=1 \mu \mathrm{m}$. 
desplazamiento de los extremos de alrededor de una altura cingular (Figs. 3C-D). Está bordeado por aletas cingulares poco prominentes, originadas en las placas adcingulares. Las suturas entre las placas cingulares están alineadas con las suturas de las placas postcingulares, exceptuando la 1C. Dicha placa es de pequeño tamaño (Figs. 3C, 4) y también podría ser interpretada como una placa transicional (t). El sulcus no penetra en la epiteca y se extiende hasta el antápice (Figs. 3C-D). Se pudo distinguir 4 placas, siendo la sulcal posterior ( $\mathrm{Sp}$ ) la de mayor tamaño y tiene contacto con la primera cingular $(1 \mathrm{C}=\mathrm{t})$ (Fig. 4). Esta última, también tiene contacto con la sulcal anterior (Sa) y sulcal izquierda (Ss). La sulcal izquierda está cubierta parcialmente por la sulcal derecha (Sd) y la sulcal anterior (Figs. 3C, 4).

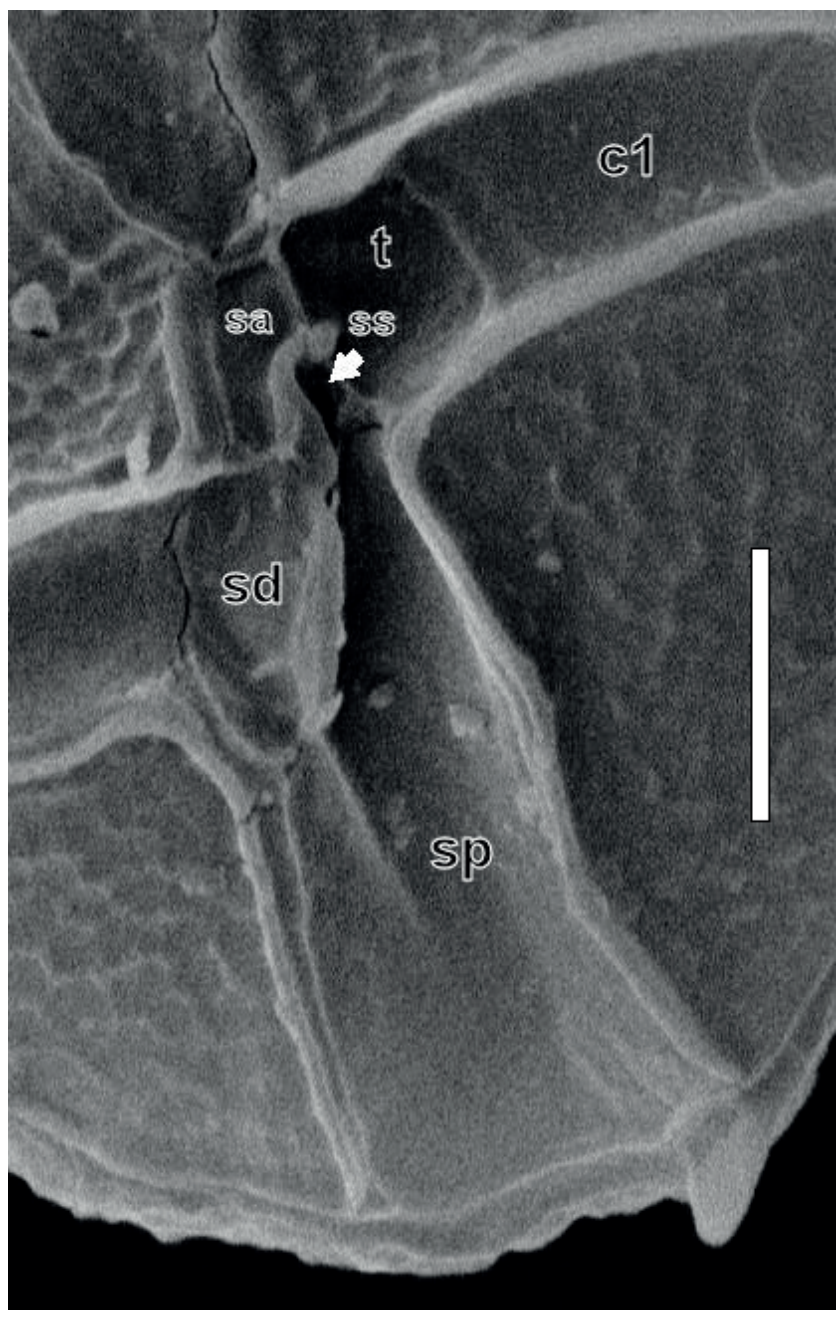

Figura 4: Naiadinium polonicum (MEB). Detalle del sulcus, $\mathrm{Sp}$ es larga y tiene contacto con el cingulum. Escala $=5 \mu \mathrm{m}$. $/$ Naiadinium polonicum (SEM). Detail of the sulcus, Sp is long and contacted with the cingulum. Scale bar $=5 \mu \mathrm{m}$.
ECOLOGÍA Y FLORA ACOMPAÑANTE

Los parámetros ambientales en superficie en el momento de recolectar el material fueron: temperatura de 25,06 ${ }^{\circ} \mathrm{C}$, $\mathrm{pH}$ de 9,16 , conductividad de $0,474 \mathrm{mS} / \mathrm{cm}$, oxígeno disuelto de $11,18 \mathrm{mg} / \mathrm{L}$, clorofila a $9 \mu \mathrm{g} / \mathrm{L}$, transparencia Secchi de $65 \mathrm{~cm}$, nitrógeno total de 645,67 $\mu \mathrm{g} / \mathrm{L}$ y fósforo total de $66 \mu \mathrm{g} / \mathrm{L}$. Naiadinium polonicum alcanzó los 2572 individuos $/ \mathrm{mL}$, siendo la especie dominante en la muestra analizada $(55,7 \%)$. Otras algas acompañantes presentes en abundancia fueron Peridinium willei Huitfeld-Kaas (13,9\%), Cryptomonas sp. (5,6\%), Euglena sp. (4,9\%), Scenedesmus sp. (4,9\%), Cyclotella meneghiniana Kützing $(3,7 \%)$, Gonatozygon sp. $(3,0 \%)$, Ceratium furcoides (Levander) Langhans (1,4\%) y Oocystis sp. (1,2\%).

\section{DISCUSIÓN}

Nuestros ejemplares concuerdan con las descripciones conocidas en la literatura para Naiadinium polonicum, citado como Peridinium polonicum o Peridiniopsis polonica (Popovský \& Pfiester 1990, Hansen \& Flaim 2007, Craveiro et al. 2015). Los individuos aquí estudiados presentaron 6 placas cingulares $(6 \mathrm{Co} 5 \mathrm{C}+\mathrm{t})$, coincidiendo con los resultados encontrados por Hansen \& Flaim (2007) y Craveiro et al. (2015), entre otros. No obstante, Carty (2014: p. 223) al establecer el género indica la presencia de cinco placas cingulares, posiblemente interpretando la $1 \mathrm{C}(=\mathrm{t})$ como una placa sulcal. La especie es variable en relación al número y forma de las placas intercalares; en nuestros ejemplares la primera intercalar anterior (1a) siempre presentó menor tamaño que la segunda intercalar (2a).

Carty (2014) distingue a $N$. polonicum y $N$. biscutelliforme por la diferencia en el número de placas intercalares anteriores. Nosotros consideramos que dicha diferencia no es significativa puesto que no va acompañada de otras diferencias morfológicas ni ecológicas. La mayoría de los investigadores que estudiaron la especie han ilustrado individuos con dos placas intercalares y habitualmente con la primera intercalar reducida (e.g., nuestros ejemplares, Imamura \& Fukuyo 1990, Roset et al. 2002, Hansen \& Flaim 2007, Hu et al. 2008, Krakhmalny 2014, Craveiro et al. 2015). En cambio, los hallazgos de tecas con una placa intercalar anterior (Wołoszyńska 1916, Popovský $1968,1970)$ o con dos placas intercalares de similar tamaño (Thompson 1951, Carty 2014) han sido poco frecuentes y sin indicación del número de ejemplares analizados. Adachi (1965) analizó una población de P. polonicum en el lago Sagami (Japón), donde observó ejemplares con epitecas que exhiben una sola placa intercalar e individuos con dos intercalares de similar tamaño, y una serie de ejemplares intermedios: epitecas con dos intercalares y con 1a reducida e individuos con la 2a reducida, esto indica un continuum entre los ejemplares con una y dos placas intercalares de 
similar tamaño. Por lo anterior, consideramos que $N$. biscutelliforme es un sinónimo de la especie $N$. polonicum que debería considerarse como una especie que en la epiteca posee un número variable de placas intercalares anteriores. En uno de los extremos del rango están los ejemplares que exhiben una placa intercalar rectangular o cuadrada (morfotipo N. polonicum según Carty 2014), en el otro extremo de variación, aquellos individuos cuyas tecas presentan dos placas intercalares anteriores de similar tamaño (morfotipo N. biscutelliforme según Carty 2014).

La tabulación general es uno de los criterios taxonómicos más importantes en la circunscripción de los dinoflagelados tecados (Bourrelly 1970, Abé 1981, Popovský \& Pfiester 1990, Carty 2014). Sin embargo, en ocasiones se sobrestima el valor taxonómico del número de placas sin considerar las variaciones tabulares (número y ordenación de las placas). En este sentido, la diferencia observada en el número de placas que presenta $N$. polonicum puede atribuirse a eventos de "tabulatio simplex-complexum" (Lindemann 1919), fenómeno de variación intraespecífica conocida también en otras especies de dinoflagelados (Lefèvre 1932, Abé 1981, Netzel \& Dürr 1984, Da et al. 2004, Sekida et al. 2011). Las variaciones en la forma de los individuos y las relaciones entre las placas, especialmente en la zona dorsal de la epiteca, son más frecuentes en los dinoflagelados de ecosistemas continentales que en los representantes marinos (Balech 1963, Boltovskoy 1983, Elbrächter \& Meyer 2001). Muchas de estas variaciones tabulares son halladas en pocos ejemplares de una población, por ende, interpretadas en la mayoría de los casos como teratologías (Lefèvre 1932, Boltovskoy 1973, Balech 1974).

Naiadinium polonicum presenta la particularidad de poseer la placa sulcal posterior (Sp) en contacto con el cingulum por medio de la primera cingular (1c) (o placa transicional ( $\mathrm{t}$ )) (este estudio: Fig. 4, Craveiro et al. 2015: figs. 13-14), característica que la relaciona con algunas especies marinas de Scrippsiella. Las especies Scrippsiella precaria Montresor \& Zingone, Scrippsiella ramonii Montresor \& Zingone y Scrippsiella irregularis Attaran-Fariman \& Bolch presentan epitecas asimétricas y el peculiar contacto entre la primera placa intercalar (1a) y la tercera intercalar (3a), y en función de análisis filogenéticos están estrechamente relacionadas con Naiadinium polonicum (Gottschling et al. 2005, 2012, Logares et al. 2007). Por ello, Craveiro et al. (2015) sugiere la necesidad de efectuar mayores estudios, basados en análisis de caracteres ultraestructurales y genéticos, que apoyen la transferencia de estas especies de Scrippsiella al género Naiadinium.

En el Embalse Rapel la especie sólo fue encontrada en una ocasión (mayo de 2013). Las elevadas cantidades de nitrógeno y fósforo total $(645,07 \mu \mathrm{g} / \mathrm{L}$ y $66 \mu \mathrm{g} / \mathrm{L}$, respectivamente) presentes en la columna de agua y las altas temperaturas $\left(25,06{ }^{\circ} \mathrm{C}\right.$ ), inusuales para los meses de otoño (Vila et al. 2000), fueron probablemente los factores preponderantes que desencadenaron la floración. Estas variables son las más señaladas en la literatura como causante de los crecimientos explosivos en los dinoflagelados (Pollingher 1987, Reynolds 2006, Almanza et al. 2016a). La calidad de agua del Embalse Rapel se ha visto deteriorada en gran medida por factores antropogénicos y en relación con el incremento de nutrientes, la biodiversidad planctónica, en especial el fitoplancton, ha experimentado cambios en la estructura comunitaria señalando un incremento de las cianobacterias Microcystis aeruginosa (Kützing) Kützing y Anabaena sp., especies típicas de los sistemas eutrofizados (Parra et al. 1980, Dorador et al. 2007, Almanza et al. 2016b). La marcada condición eutrofizada del embalse que ha sido señalada en estudios previos (Vila et al. 2000, Vila \& Pardo 2003, Martínez et al. 2003) y nuestros propios datos, concuerdan con las condiciones encontradas en floraciones de $N$. polonicum en el lago Tenkiller Ferry (Oklahoma, Estados Unidos), donde los eventos masivos fueron asociados a los altos niveles de nitrógeno presentes en los sitios de muestreo (Nolen et al. 1989). En cambio, los valores de $\mathrm{pH}$ y temperatura medidos en nuestro estudio son más elevados a los mencionados en las proliferaciones masivas reportadas en España (pH: 7,64-8, T: 18-22 ${ }^{\circ} \mathrm{C}$; Roset et al. 2002).

La información disponible en relación a las preferencias tróficas de la especie es escasa. Hansen \& Flaim (2007) la encuentran en los meses de primavera y verano en tres lagos italianos de condiciones mesotróficas y eutróficas, con concentraciones de fósforo total entre 11-35 $\mu \mathrm{g} / \mathrm{L}$. En el lago Kinneret, un cuerpo de agua eutrófico subtropical, apareció esporádicamente en bajas cantidades en la zona pelágica, pero muchas veces fue abundante en la zona litoral (Pollingher \& Hickel 1991). En el lago Boondoggle (Estados Unidos) la especie fue encontrada en mayor número de individuos durante los meses de verano, donde el lago presenta estratificación del oxígeno y altas temperaturas (Canion \& Ochs 2005). Nuestros hallazgos tienden a confirmar la preferencia de la especie por las aguas cálidas y con altas concentraciones de nutrientes. Sin embargo, tanto los antecedentes de la literatura como nuestros datos deben tomarse como una información parcial, ya que son hallazgos puntuales.

La especie ha sido reportada en numerosas localidades de Europa (Penard 1891, Wołoszyńska 1916, Lefèvre 1932, Popovský 1968, Bourrelly \& Couté 1980, Roset et al. 2002, Hansen \& Flaim 2007, Krakhmalny 2014, Craveiro et al. 2015), Asia (Adachi 1965, Imamura \& Fukuyo 1990, Pollingher \& Hickel 1991, Krakhmalny et al. 2000; Hu et al. 2008), Norteamérica (Osorio-Tafall 1941, Thompson 1951, Carty 2014) y Centroamérica (Popovský 1970). En América del Sur ha sido reportada en el Embalse Serraria (Estado de São Paulo, Brasil) como Glenodinium gymnodinium (Sant'Anna et al. 1988). En el catálogo del fitoplancton de aguas continentales de Chile se menciona a Glenodinium gymnodinium sin indicar localidad ni fecha de prospección 
(Parra et al. 1982: p. 35). Sin embargo, no hemos encontrado para Chile mención ni iconografía atribuible a la especie en estudio. En consecuencia, consideramos que el presente estudio reporta a $N$. polonicum por primera vez para Chile, siendo el Embalse Rapel la localidad geográfica más austral encontrada hasta el presente, ampliando la distribución mundial de la especie. El conocimiento de los dinoflagelados presentes en los ecosistemas acuáticos continentales chilenos es limitado, los reportes son escasos y en su mayoría no proporcionan ilustraciones ni diagnosis de los taxa (Parra \& González 1977, Parra 2006) y las investigaciones descriptivas son recientes (Ascencio et al. 2015, Ascencio 2017), siendo necesario realizar esfuerzos en conocer la diversidad de los dinoflagelados del país, cuya riqueza específica está subestimada y sus relaciones ecológicas desconocidas.

En Chile han sido mencionados desarrollos masivos de los dinoflagelados invasores Ceratium hirundinella (O.F.Müller) Dujardin (Parra et al. 1991: p. 105, Parra 1998) y de C. furcoides (Almanza et al. 2016a, Ascencio 2017). La mayoría de la información de proliferaciones algales generadas en Chile se restringen a eventos producidos por cianobacterias (Parra et al. 1980, 1986, Campos et al. 2005, Almanza et al. 2016b), diatomeas bentónicas (Rivera et al. 2013, Olivares et al. 2015, Basualto et al. 2016) у en menor frecuencia especies de euglenófitas y clorófitas (Almanza et al. 2016c). Por lo anterior, la presencia masiva de $N$. polonicum detectada en este estudio agrega una nueva potencial alga nociva en los ecosistemas acuáticos continentales del país. Por ende, se requiere monitorear la dinámica de la taxocenosis del Embalse Rapel para determinar la eventual repetición y periodicidad de estos fenómenos con la finalidad de evaluar los potenciales efectos en el recurso hídrico.

\section{AGRADECIMIENTOS}

Agradecemos al programa de observación del ambiente litoral (POAL) de la Armada de Chile y a los Drs. Susan Carty (Ohio, USA), Santiago Fraga (Vigo, España), Alexander F. Krakhmalny (Kiev, Ucrania), Carlos E.M. Bicudo (São Paulo, Brasil) y Kaoli P. Cavalcante (Sobral, Brasil) por el envío de material bibliográfico de difícil acceso y al Dr. Yuri B. Okolodkov (Veracruz, México) por la traducción de textos rusos. Apreciamos los comentarios taxonómicos realizados por la Dra. Sandra C. Craveiro y António J. Calado (Aveiro, Portugal) y al Dr. Andrés Boltovskoy (La Plata, Argentina) por la revisión crítica del manuscrito. Reconocemos la asistencia técnica del personal del Centro de Espectroscopia y Microscopia Electrónica de la Universidad de Concepción.

\section{REFERENCIAS}

ABÉ, T.H. 1981. Studies on the family Peridiniidae. An unfinished monograph of the armoured dinoflagellate. Publications of the Seto Marine Biological Laboratory, Special Publication Series 6: 1-409.

ADACHI, R. 1965. Studies on a dinoflagellate Peridinium polonicum Woloszynska I. The structure of skeleton. Journal of Faculty of Fisheries, Prefectural University of Mie 6(3): 317-326.

Almanza, V., Bicudo, C.E.M., Parra, O., Urrutia, R. 2016 a. Características morfológicas y limnológicas de las floraciones de Ceratium furcoides (Dinophyta) en un lago somero de Chile central. Limnetica 35(1): 253-268.

Almanza, V., Parra, O., Bicudo, C.E.M., Baeza, C., Beltran, J., Figueroa, R., Urrutia, R. 2016b. Occurrence of toxic blooms of Microcystis aeruginosa in a central Chilean $\left(36^{\circ}\right.$ Lat. S) urban lake. Revista Chilena de Historia Natural 89: 8. http://dx.doi.org/10.1186/s40693-016-0057-7

Almanza, V., Parra, O., Bicudo, C.E.M., GonzÁlez, M.A., Lopez, M., Urrutia, R. 2016c. Floraciones de fitoplancton y variaciones de la estructura comunitaria fitoplanctónica en tres lagos someros eutróficos de Chile central. Gayana Botánica 73(2): 191-205.

Ascencio, E. 2017. Diversidad de los dinoflagelados tecados presentes en dos lagos urbanos de Concepción (VIII Región), Chile. Tesis de Biólogo. Facultad de Ciencias Naturales y Oceanográficas, Universidad de Concepción. Concepción, Chile. 51 pp.

Ascencio, E., Rivera, P., Cruces, F. 2015. Morfología de Peridiniopsis elpatiewskyi (Ostenfeld) Bourrelly (Dinophyceae) encontrada por primera vez en aguas continentales de Chile. Gayana Botánica 72(1): 42-46.

BAlech, E. 1963. Dos dinoflagelados de una laguna salobre de la Argentina. Notas del Museo de La Plata 20, Zoología 199: 111-123.

BAlech, E. 1974. El género Protoperidinium Bergh, 1881 (Peridinium Ehrenberg, 1831, partim). Revista del Museo Argentino de Ciencias Naturales "Bernardino Rivadavia", Hidrobiología 4(1): 1-79.

Basualto, S., Rivera, P., Cruces, F., Ector, L., Ascencio, E. 2016. Didymosphenia geminata (Lyngbye) M. Schmidt y Gomphoneis minuta (Stone) Kociolek \& Stoermer (Bacillariophyta), especies presentes en ríos de la VIII Región, Chile, formadoras de proliferaciones con aspecto muy similar. Gayana Botánica 73(2): 457-461.

Boltovskoy, A. 1973. Peridinium gatunense Nygaard. Estructura y estereoultraestructura tecal (Dinoflagellida). Physis B 32(85): 331-344.

Boltovskoy, A. 1983. Peridinium cinctum f. westii del mar de Galilea, sinónimo de Peridinium gatunense (Dinophyceae). Limnobios 2(6): 413-418.

Boltovskoy, A. 1995. Taxonomía y morfología de los dinoflagelados: Métodos de trabajo. En: Alveal, K., Ferrario, M.E, Oliveira, E.C., Sar, E. (eds.), Manual de Métodos Ficológicos, pp. 55-82. Universidad de Concepción, Concepción, Chile.

Bourrelly, P. 1968. Notes sur les Péridiniens d'eau douce. Protistologica 4(1): 5-16.

Bourrelly, P. 1970. Les algues d'eau douce. Tome III: Les 
algues bleues et rouges. Les Eugléniens, Peridiniens et Cryptomonadines. Éditions N. Boubée \& Cie, Paris. 512 pp.

Bourrelly, P., Couté, A. 1980. Trois Dinophycées d'eau douce observées en microscopie a balayage. Cryptogamie Algologie 1(2): 161-169.

Campos, V., Lisperguer, S., Weckesser, J., Vera, A., Muñoz, D. 2005. Cianobacterias y riesgos potenciales de toxicidad en aguas continentales de Chile. Boletín Micológico 20: 73-81.

CAnion, A.K., Ochs, C. 2005. The population dynamics of freshwater armored dinoflagellates in a small lake in Mississippi. Journal of Freshwater Ecology 20(4): 617626.

CARTY, S. 2014. Freshwater dinoflagellates of North America. Comstock Publishing Associates, Ithara. 260 pp.

Craveiro, S.C., Daugbuerg, N., Moestrup, Ø., Calado, A.J. 2015. Fine-structural characterization and phylogeny of Peridinium polonicum, type species of the recently described genus Naiadinium (Dinophyceae). European Journal of Protistology 51(4): 259-279.

Da, K.P., Zongo, F., Mascarell, G., CoutÉ, A. 2004. Bagredinium, un nouveau genre de Péridiniales (Dinophyta) d'eau douce de l'Afrique de l'Ouest. Archiv für Hydrobiologie/ Supplement 150, Algological Studies 111: 45-61.

Dorador, C., Castillo, G., Wetzel, K.P., Vila, I. 2007. Bacterial diversity in the sediments of a temperate artificial lake Rapel reservoir. Revista Chilena de Historia Natural 80(2): 213-224.

Elbrächter, M., Meyer, B. 2001. Plate pattern variability and plate overlap in a clonal culture of the freshwater dinoflagellate Peridinium umbonatum Stein species complex (Dinophyceae). Neues Jahrbuch für Geologie und Paläontologie Abhandlungen 219(1/2): 221-227.

Gottschling, M., Knop, R., Plötner, J., Kirsch, M., Willems, H., Keupp, H. 2005. A molecular phylogeny of Scrippsiella sensu lato (Calciodinellaceae, Dinophyta) with interpretations on morphology and distribution. European Journal of Phycology 40(2): 207-220.

Gottschling, M., Soehner, S., Zinssmeister, C., John, U., Plötner, J., Schweikert, M., Aligizaki, K., Elbrächter, M. 2012. Delimitation of the Thoracosphaeraceae (Dinophyceae), including the calcareous dinoflagellates, based on large amounts of ribosomal RNA sequence data. Protist 163(1): 15-24.

Hansen, G., Flaim, G. 2007. Dinoflagellates of the Trentino Province, Italy. Journal of Limnology 66(2): 107-141.

Hashimoto, Y., Okaichi, T., Dang, Le D., Noguchi, T. 1968. Glenodinine, an ichthyotoxic substance produced by a dinoflagellate, Peridinium polonicum. Bulletin of the Japanese Society of Scientific Fisheries 34(6): 528-534.

Hu, S., Liu, G.X., Zhou, G.J., Mer, H., Hu, Z.Y. 2008. Peridinium polonicum, a new record of freshwater toxic dinoflagellate from China. Journal of Wuhan Botanical Research 26(5): 454-457.

Huber- Pestalozzi, G. 1950. Das Phytoplankton des Süßwassers. Cryptophyceen, Chloromonadinen, Peridinieen. In: Thienemann, A. (ed.), Die Binnegewässer einzeldarstellungen aus der limnologie und ihren nachbargebieten 16(3), pp. 1-310. E. Schweizerbart'sche Verlagsbuchhandlung, Stuttgart, Deutschland.
Imamura, K., FukuYo, Y. 1990. Peridinium polonicum Wołoszyńska. In: Fukuyo, Y., Takano, H., Chihara, M., Matsuoka, K. (eds.), Red tide organisms in Japan. An illustrated taxonomic guide, pp. 136-137. Uchida Rakakuho, Tokyo, Japan.

Kiselev, I.A. 1954. Pyrrophyte algae. Manual for identification of freshwater algae of the USSR 6. Sovetskaya Nauka, Moskva. 212 pp.

Krakhmalny, A.F. 2014. Dinophyta of Denyshi reservoir (Zhytomyr region, Ukraine). In: V International conference "Actual problems in modern phycology", pp. 187-193. Academy of Science of Moldova, Chisinau, Moldova.

Krakhmalny, A.F., Wasser, S.P., Nevo, E. 2000. Dinophyta. In: Nevo, E., Wasser, S.P. (eds.), Biodiversity of Cyanoprocaryotes, algae and fungi of Israel: Cyanoprocaryotes and algae of continental Israel, pp. 177183. A.R.G. Gantner Verlag K.G., Ruggell, Germany.

LefÈvre, M. 1932. Monographie des espèces d'eau douce du genre Peridinium Ehrb. Archives de Botanique 2(5): 1-210.

Lindemann, E. 1919. Untersuchungen über Süßwasserperidineen und ihre variationsformen. Archiv für Protistenkunde 39: 209-262.

Lindemann, E. 1925. Peridineen des oberrheins und seiner altwässer. Botanisches Archiv 11: 474-481.

Logares, R., Shalchian-Tabrizi, K., Boltovskoy, A., Rengefors, K. 2007. Extensive dinoflagellate phylogenies indicate infrequent marine-freshwater transitions. Molecular Phylogenetics and Evolution 45: 887-903.

Martínez, G., Contreras, M., Vila, I. 2003. Modelos conceptuales de abundancia de fitoplancton asociados a la heterogeneidad espacial en el Embalse Rapel (Chile central). Revista Chilena de Historia Natural 76(2): 255-266.

Matvienko, O.M., Litvinenko, R.M. 1977. Pyrrophyta. Identification manual of freshwater algae of Ukrainian SSR 3(2). Naukova Dumka, Kiev. 386 pp.

McNeill, J., Barrie, F.R., Buck, W.R., Demoulin, V., Greuter, W., Hawksworth, D.L., Herendeen, P.S., Knapp, S., Marhold, K., Prado, J., Prud'Homme van Reine, W.F., Smith, G.F., Wiersema, J.H., Turland, N.J. 2012. International Code of Nomenclature for algae, fungi, and plants (Melbourne Code) adopte by the Eighteenth International Botanical Congress Melbourne, Australia, July 2011. Regnum vegetabile 154. Koeltz Scientific Book, Königstein. 240 pp.

Mühlhauser, H.A., Soto, L., Zahradnik, P. 1987. Improvement of the Kjeldahl method for total nitrogen including acidhydrolyzable phosphorus determinations in freshwater ecosystems. International Journal of Environmental and Analytical Chemistry 28 (3): 215-226.

Netzel, H., DürR, G. 1984. Dinoflagellate cell cortex. In: Spector, D.L. (ed.), Dinoflagellates, pp. 43-105. Academic Press, Florida, USA.

Nolen, S.L., Carroll, J.H., Combs, D.L., Staves, J.C., Veenstra, J.N. 1989. Limnology of Tenkiller Ferry Lake, Oklahoma, 1985-1986. Proceedings of the Oklahoma Academy of Science 69: 45-55.

Olivares, P., Orellana, P., Guerra, G., Peredo-Parada, M., Chavez, V., Ramirez, A, Parodi, J. 2015. Water contaminated with Didymosphenia geminata generates changes in Salmo salar spermatozoa activation times. Aquatic Toxicology 163: 102-108.

Oshima, Y., Minami, H., Takano, Y., Yasumoto, T. 1989. 
Ichtyotoxins in a freshwater dinoflagellate Peridinium polonicum. In: Okaichi, T., Anderson, D.H., Nemoto, T. (eds.), Red Tides: Biology Environmental Science and Toxicology. Proceedings of the First International Symposium on Red Tides, pp. 375-378. Elsevier Press. New York, USA.

Osorio-Tafall, B.F. 1941. Materiales para el estudio del microplancton del lago Pátzcuaro(México)I. Generalidades y fitoplancton. Anales de la Escuela Nacional de Ciencias Biológicas 2(2): 331-383.

PARRA, O. 1998. Una aproximación sistémica para la evaluación de la biodiversidad algal en ambientes acuáticos continentales de Chile. Sociedade Ficológica da America Latina e Caribe, Sociedade Brasileira de Ficologia I: 167-178.

PARRA, O. 2006. Estado de conocimiento de las algas dulceacuícolas de Chile (excepto Bacillariophyceae). Gayana 70(1): 8-15.

Parra, O., Avilés, D., Becerra, J., Dellarossa, V., Montoya, R. 1986. First toxic blue-green algal bloom recorded for Chile: a preliminary report. Gayana Botánica 43(1-4): 15-17.

Parra, O., GonzÁlez, M. 1977. Catálogo de las algas dulceacuícolas de Chile. Pyrrophyta, Chrysophyta-Chrysophyceae, Chrysophyta-Xanthophyceae, Rhodophyta, Euglenophyta y Chlorophyta. Gayana Botánica 33: 1-102.

Parra, O., González, M., Dellarossa, V., Rivera, P., Orellana, M. 1982. Manual taxonómico del fitoplancton de aguas continentales con especial referencia al fitoplancton de Chile. III. Cryptophyceae, Dinophyceae, Euglenophyceae. Universidad de Concepción, Concepción. 99 pp +21 láminas.

Parra, O., Rivera, P., Floyd, G.L, Wilcox, L.W. 1991. Cultivo, morfología, ultraestructura y taxonomía de un fitoflagelado asociado a mareas rojas en Chile: Heterosigma akashiwo (Hada) Hada. Gayana Botánica 48(1-4): 101-110.

Parra, O., Ugarte, E., Mora, S., Liberman, M., Aron, A., Balanoff, L. 1980. Remarks on a bloom of Microcystis aeruginosa Kützing. Nova Hedwigia 33: 971-1004.

Penard, E. 1891. Les Péridiniacées du Léman. Bulletin des Travaux de la Société Botanique de Géneve 6: 1-63.

Pollingher, U. 1987. Freshwater ecosystems. In: Taylor, F.J.R. (ed.), The biology of Dinoflagellates. Botanical Monograph 21, pp. 502-529. Blackwell Scientific Publication, Oxford, USA.

Pollingher, U., Hickel, B. 1991. Dinoflagellate associations in a subtropical lake (Lake Kinneret, Israel). Archiv für Hydrobiologie 120(3):267-285.

Popovský, J. 1968. A contribution to the knowledge of dinoflagellates from Bohemia. Preslia 40: 251-263.

Popovský, J. 1970. Some thecate dinoflagellates from Cuba. Archiv für Protistenkunde 112: 252-258.

Popovský, J., Pfiester, L.A. 1990. Dinophyceae (Dinoflagellida). In: Ettl, H., Gerloff, J., Heynig, H., Mollenhauer, D. (eds.), Süßwasserflora von Mitteleuropa 6, pp. 1-271. Gustav Fischer Verlag, Jena, Germany.

Rengefors, K., Legrand, C. 2001. Toxicity in Peridinium aciculiferum- an adaptive strategy to outcompete other winter phytoplankton? Limnology and Oceanography 46(8): 1990-1997.

ReYNOLDS, C.S. 2006. The ecology of phytoplankton. Cambridge University Press, New York. 535 pp.
Rivera, P., Basualto, S., Cruces, F. 2013. Acerca de la diatomea Didymosphenia geminata (Lyngbye) M. Schmidt: su morfología y distribución en Chile. Gayana Botánica 70(1): 154-158.

Roset, J., Gibello, A., Aguayo, S., Domínguez, L., Álvarez, M., Fernández-Garayzabal, J.F., Zapata, A., Muñoz, M.J. 2002. Mortality of rainbow trout [Oncorynchus mykiss (Walbaum)] associated with freshwater dinoflagellate bloom [Peridinium polonicum (Woloszynska)] in a fish farm. Aquaculture Research 33(2): 141-145.

SAnt'Anna, C.L., Xavier, M.B., Sormus, L. 1988. Estudo qualitativo do fitoplâncton da represa de Serraria, Estado de São Paulo, Brasil. Revista Brasileira de Biologia 48(1): 83-102.

SCHILLER, J. 1937. Dinoflagellatae (Peridineae) in monographischer behandlung. In: R. Kolkwitz (ed.), Rabenhorst's Kryptogamen-Flora von Deutschland, Österreich und des Schweiz 10(3), pp. 1-589. Akademische Verlagsgesellaschaft, Leipzig, Deutschland.

Sekida, S., Takahira, M., Horiguchi, T., Okuda, K. 2011. Effects of high pressure in the armored dinoflagellate Scrippsiella hexapraecingula (Peridiniales, Dinophyceae): changes in thecal plate pattern and microtubule assembly. Journal of Phycology 48: 163-173.

Starmach, K. 1974. Cryptophyceae, Dinophyceae, Raphidophyceae. In: Starmach, K, Siemińska, J. (eds.), Flora Słodkowodna Polski 4, pp. 1-520. Państwowe Wydawnictwo Naukowe. Warszwa, Kraków, Poland.

Thompson, R. H. 1951. A new genus and new records of freshwater Pyrrophyta in the Desmokontae and Dinophyceae. Lloydia 13(4): 277-299.

VILA, I., PARDO, R. 2003. Respuesta de la estructura fitoplanctónica a las perturbaciones antrópicas de un lago temperado. Limnetica 22(1-2): 93-102.

Vila, I., Contreras, M., Montecino, V., Pizarro, J., Adams, D.D. 2000. Rapel: a 30 years temperate reservoir. Eutrophication or contamination? Archiv für Hydrobiologie, Special Issues Advances in Limnologie 55: 31-44.

ViLlafañe, V.E., ReId, F.M.H. 1995. Métodos de microscopia para la cuantificación del fitoplancton. En: Alveal. K., Ferrario, M.E., Oliveira, E.C., Sar, E. (eds.), Manual de métodos ficológicos, pp. 169-185. Universidad de Concepción, Concepción, Chile.

WHO. 2003. Guidelines for safe recreational water environments. Volume 1: coastal and fresh waters. World Health Organization, Geneva. 253 pp.

WoŁoszyńska, J. 1916. Polnische süßwasser-peridineen. Bulletin de L'Académie des Sciences de Cracovie. Classe des Sciences Mathématiques et Naturelles, Série B Sciences Naturelles (1915): 260-285, 10-14 planches.

Wu, J.T., Kuo-Huang, L.L., Lee, J. 1998. Algicidal effect of Peridinium bipes on Microcystis aeruginosa. Current Microbiology 37(4): 257-261.

Zaccaroni, A., Scaravelli, D. 2008. Toxicity of freshwater algal toxins to humans and animal. In: Evangelista, V., Barsanti, L., Frassanito, A.M., Passarelli, V., Gualtieri, P. (eds.), Algal toxins: nature, occurrence, effect and delection, pp. 45-89. NATO science for peace and security series A: chemistry and biology, Dordrecht, The Netherlands.

Recibido: 11.09.2017

Aceptado: 15.12.2017 